\title{
Research Interconnection of Information for Digital Workshop of Machine Tools Manufacturing
}

\author{
Kaiyu Song ${ }^{1, a}$, Min Wang ${ }^{1,2, b}$ \\ ${ }^{1}$ Beijing University of Technology, Beijing 100124, China \\ ${ }^{2}$ Beijing Municipal Key Laboratory of electrical discharge machining technology, Beijing 100191, \\ China. \\ a kaiyusong@163.com, ${ }^{b}$ wangm@bjut.edu.cn
}

Keywords: Intelligent Manufacturing, Information Interconnection, Information Integration.

\begin{abstract}
With the popularization and rapid development of network, intelligent, networked and green manufacturing has gradually become an important direction of development. Improving the intellectualization of digital workshop of machine tools manufacturing (DWMTM) will become the trend of machine tools manufacturing enterprises in the future. On the basis of introducing the status quo of information integration in digitized workshop of domestic backbone machine tools manufacturing enterprises, the problems of information interconnection and information integration in DWMTM at present are put forward. According to the requirement of intelligence, the network architecture, information exchange mechanism and information flow in the DWMTM are analyzed and studied.
\end{abstract}

\section{Introduction}

With the popularization and rapid development of information technology and the Internet, the advent of the network economy era has brought about tremendous changes in the external environment of the manufacturing industry [1-3]. Machine tools are the mother machine of manufacturing industry, the carrier of advanced manufacturing technology and the basic means of production of the equipment industry [4]. Machine tools manufacturing have become the basis for the development of manufacturing industry. Machine tools manufacturing technology have grown up to be an important indicator of national strength. With continuous technological progress in the fields of manufacturing and related information network control, the technology of digital workshop of machine tool manufacturing (DWMTM) is developing rapidly. The technology of DWMTM involves a wide range of professions, including industrial computers, workstations, servers, programmable controllers and other information systems, industrial robots, intelligent vision, tactile sensor systems, logistics transmission and storage systems, CNC machine tools, on-line detection systems, cutting management database systems, production Management system and other aspects are the comprehensive application of digital and network technology in machine tools manufacturing workshop. By integrating the information of $\mathrm{NC}$ equipment and process design, production 
organization system and monitoring management system, an integrated manufacturing system with integrated information flow automation is formed, which can enhance the organization of machine tools manufacturing production as a whole. And management to increase the flexibility of machine tools manufacturing workshop and the utilization ratio of equipment in the workshop.

\section{Background}

Backbone machine tools manufacturing enterprises in China have launched their own representative digital workshop, and strive to develop machine tools manufacturing technology from automation mode to an information-driven network integrated control mode. However, through the analysis, we can find that there are many kinds of information integrated management functions in the DWMTM, which covers many aspects, such as NC equipment and process design, production organization and management, and other management systems. Each machine tools manufacturer implements the above functions on the MES platform of the digitized workshop designed independently. There is no uniform standard specification for the information communication format and network architecture adopted. It is necessary to redevelop the information integration interfaces of various heterogeneous platforms in the device layer, application layer and data layer for information integration application and network data transmission, which lead to the high cost of technology development in the DWMTM. It will also make it difficult to realize effective network integration between heterogeneous software and hardware for the device and device, device and SCADA of digital workshop, which involve product information, process information, device information status information and workshop management information of machine tools manufacturing. This will further limit the realization of effective networked and Intelligent Collaborative Manufacturing in the DWMTM.

\section{Proposed Approach}

\subsection{Architecture of interconnected network}

The architecture of interconnected network. Intelligent manufacturing requires manufacturing enterprises to break the disconnection between the original business process and the production process, so that all levels of systems and equipment distributed in the production and manufacturing links are no longer "information isolated island". Information exchange requires from the bottom equipment layer up to the workshop layer or even enterprise layer network, making the workshop layer or enterprise layer. It can monitor real-time production status and equipment information, and optimize and adjust production scheduling and resource allocation according to the information obtained. According to the requirement of intelligent manufacturing, this paper presents a typical architecture of interconnected network for DWMTM, which conforms to the hierarchical definition of intelligent manufacturing system, as shown in Figure 1. The enterprise layer is not in DWMTM.

The functions of each layer are defined as follows:

- Enterprise layer: this layer realizes the management of machine tools manufacturing enterprises, it covers the management of customers, projects, inventory and procurement, supply, production and so on. This layer includes Enterprise Resource Planning system (ERP), Product Life cycle Management (PLM), Supply Chain Management system (SCM) and Customer Relationship Management system (CRM). The level of information transmission should be month, week and day. 
- Workshop layer: this layer implements production management oriented to the machine tools manufacturing workshop, this layer should include MES. The level of information transmission should be day, hour and minute.

- Control layer: it realizes the monitoring and control of machine tools manufacturing process, and defines the activities of monitoring and control of machine tools manufacturing process. This layer includes SCADA, and also including PLC, DCS and other control devices. The level of information transmission should be minutes, seconds and milliseconds.

- Device layer: this layer implements field equipment for machine tool manufacturing process. This field equipment can include various sensors, actuators, RFID, etc. It can also include various types of CNC, FMS, industrial robots, digital detection equipment, digital assembly equipment, intelligent warehousing and logistics equipment, AGV and other manufacturing equipment. The level of information transmission should be seconds, milliseconds, microseconds.

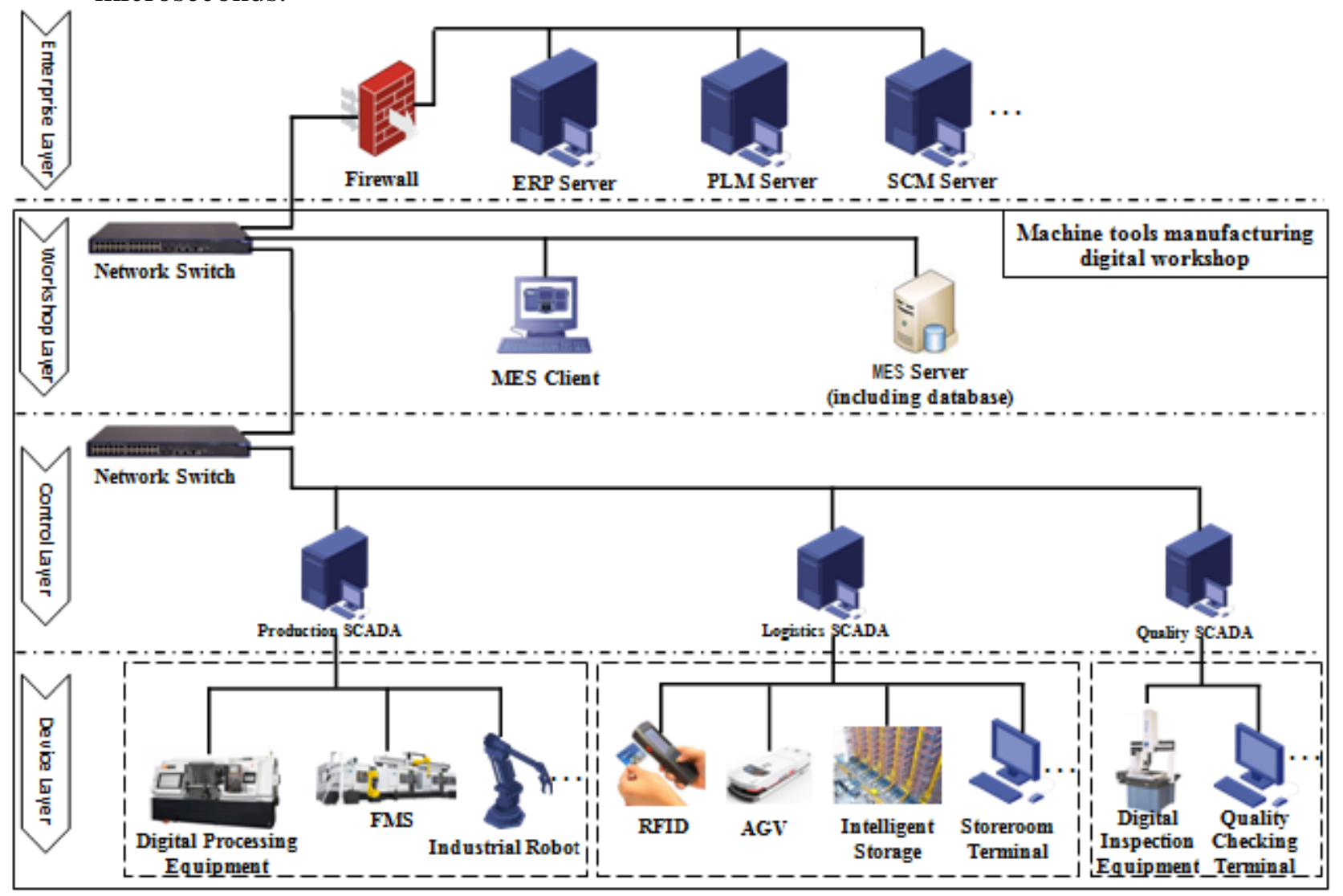

Fig. 1 Typical architecture of interconnected network for DWMTM

The advantages of the architecture are as follows:

1) The design intention of the three-tier network architecture is to adopt the idea of distributed system and distribute all the tasks involved in workshop production activities to different intelligent equipment on the whole network architecture of DWMTM to maximize the use of all the resources in the whole workshop and locate the equipment at different levels and locations. The information that each needs to process is processed to complete the corresponding tasks, and the integrated information is exchanged with the outside world to optimize the allocation of resources and improve the production efficiency of enterprises.

2) The three-tier network architecture makes the overall structure of DWMTM clear, different levels of equipment to deal with their own tasks independently, reduce the coupling between 
the layers to avoid the overall system paralysis caused by equipment failures between the layers.

3) Each machine can store and process data in the distributed network of DWMTM. It makes full use of the ability of the machine to store and process data in the distributed network, reduces the requirement for the performance of the main server machine at the management level, and can reduce the hardware cost appropriately.

\subsection{Interaction mechanism of information}

Interaction mechanism of information. In order to coordinate the prominent contradictions between the existing digital control equipment and the business management system in DWMTM, such as heterogeneous equipment and platform differences, an open and shared information exchange mechanism is needed. This paper presents an interactive mechanism of information interconnection in DWMTM based on electronic worksheet (EW) and parser, as shown in Figure 2. EW is responsible for describing the field equipment and related working procedures, entity resources and digital resources in DWMTM. The parser is not only responsible for parsing, editing, deriving and merging $\mathrm{EW}$, but also sending and receiving EW in the network.

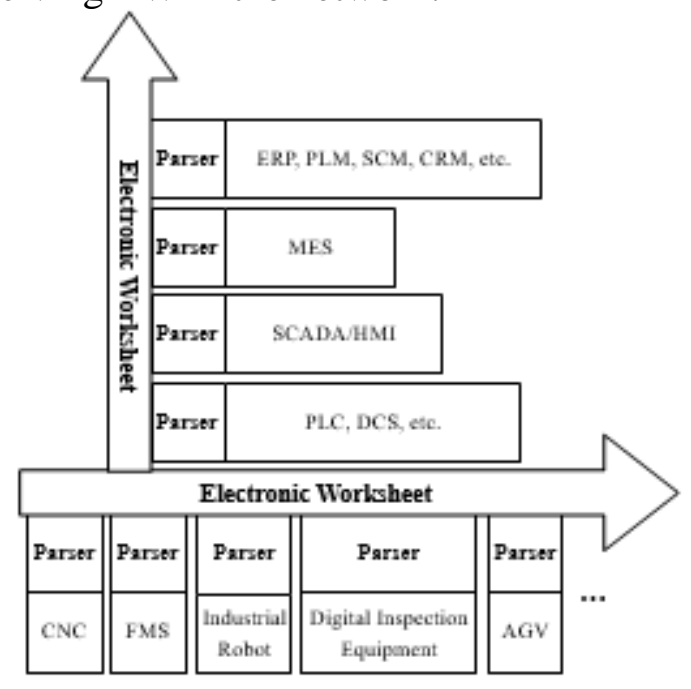

Fig. 2 Interaction mechanism of information

EW is an information exchange medium between all kinds of equipment and manufacturing execution system in the whole DWMTM. It is used to describe the data structure of machine tools products and the logical relationship of the production process organization and record the information of production management, scheduling, process, quality and inventory in the manufacturing process of machine tool parts. Definition format is the interconnected information model of DWMTM. Figure 3 shows the overall structure of the EW. As can be seen from the diagram, the EW is composed of three parts, namely, product electronic worksheet (PEW), process message electronic worksheet (PMEW) and device capability electronic worksheet (DCEW). The PEW includes the production process electronic worksheet (PPEW), the quality management electronic worksheet (QMEW) and the logistics management electronic worksheet (LMEW). 


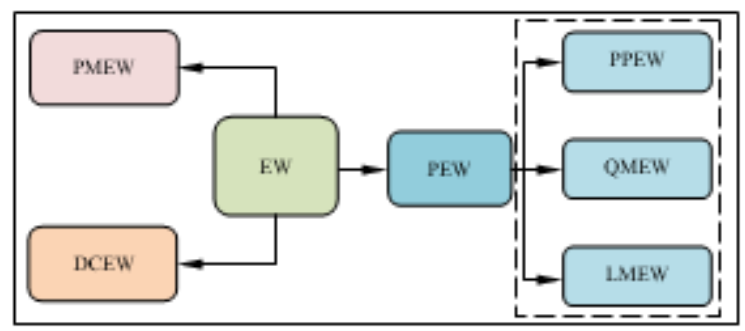

Fig. 3 The overall structure of EW

The functions of various EW are as follows:

- PEW: It is used to record the production plan of machine tool parts produced in the DWMTM.

- PPEW: It is used to record related information of machine parts manufacturing process in the DWMTM..

- QMEW: It is used to record the quality requirements of machine tools parts and related quality inspection results processed in the DWMTM.

- LMEW: It is used to record the information of inbound, outbound and transshipment of blanks, parts in process and finished products in the secondary warehouse of DWMTM.

- PMEW: It handles the message communication of the system.

- DCEW: It is used to record the description information of the field apparatus in the DWMTM. These descriptions should contain both static parameter information(for example: the spindle number, spindle speed, and the size of the worktable, etc.) and dynamic operation statistics (for example: processing time, failure time, failure rate, etc.)of the device.

\subsection{Information flow}

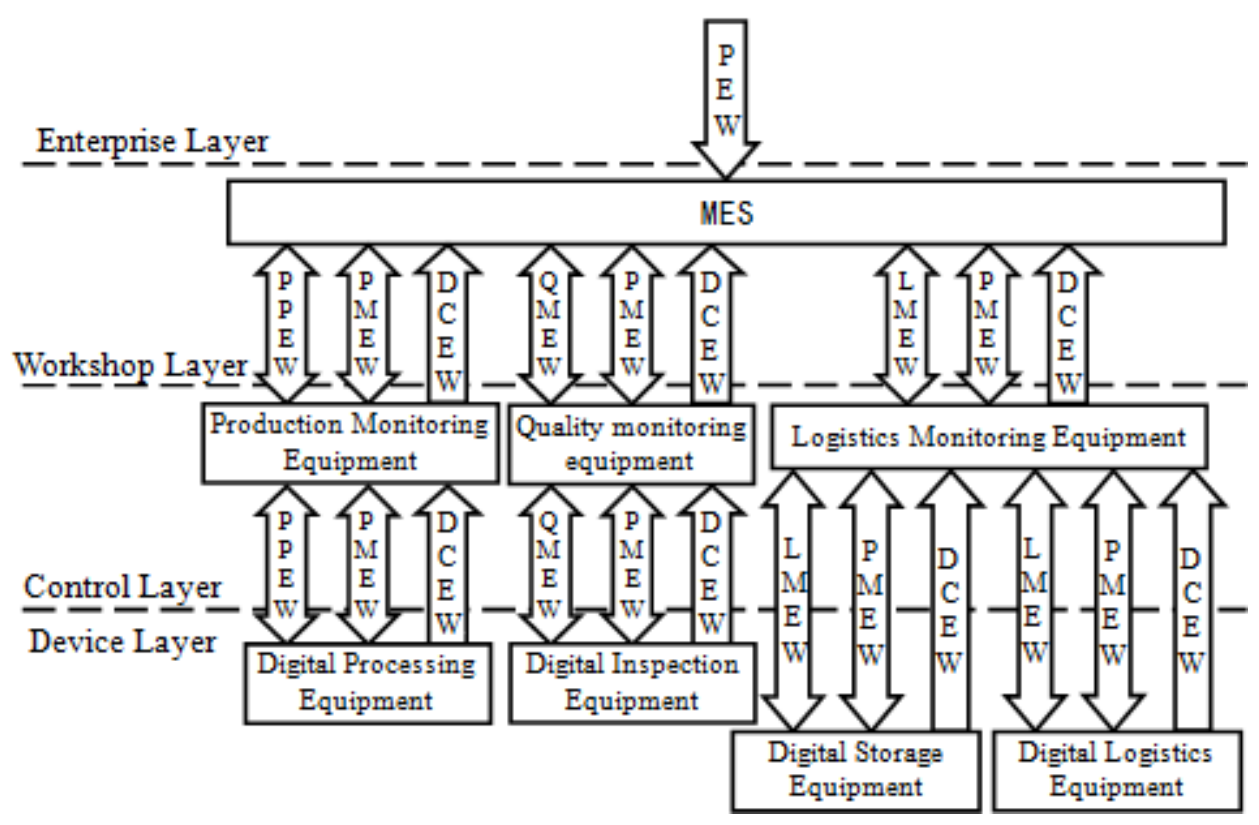

Fig. 4 Information flows in DWMTM

Figure 4 shows the possible exchange of information between the hardware and software components associated with machine tools production at each layer (arrows denote the direction of the information flow, one-way arrows denote one-way communication, and two-way arrows denote two-way communication). The enterprise layer should send the information of parts production plan to the MES of the workshop layer through the EW, the MES should send the information of 
production task and production order to the monitoring equipment of the control layer through the $\mathrm{EW}$, and the monitoring equipment of the control layer should send the production order to the field equipment of the device layer through the EW. Each monitoring equipment in the layer should upload the field data, device capability and management information after processing to MES through the EW; the field equipment in the device layer should upload the device capability, status information and other field data to the monitoring equipment in the control layer through the EW.

\section{Conclusions}

This paper first analyzes the problems existing in the production process of the DWMTM, then constructs the network architecture of the DWMTM according to the analysis of the existing problems and the requirements of intelligent manufacturing, and defines the hierarchical division of the information interconnection of the DWMTM, and on this basis establishes the network architecture of the DWMTM. An interactive mechanism based on EW and parser for information interconnection in the DWMTM is proposed. The possible exchange of information between hardware and software parts related to machine tools production at each layer is given.

\section{Acknowledgments}

This research is supported by National Natural Science Foundation of China (Grant No.51575014) and 2015 intelligent manufacturing project of ministry of industry and information technology of the People's Republic of China (Grant No.2015ZXFB02001).

\section{References}

[1] J.C. Bin, Development direction and Countermeasures of China's manufacturing industry under the new international division of labor system, Asia-pacific Economic Review. 1 (2013) 121-127.

[2] Y.Y. Wang, H.R. Zhang, Development status of global intelligent manufacturing industry and China's Countermeasures, Southeast Academic Research. 6 (2016) 116-123.

[3] J.N. Si, Equipment industry reshaped by global pattern of manufacturing industry, China Equipment. 5 (2016) $54-57$. [4] J. Li, X. Wei, T. Chen, Application of numerical control technology in mechanical manufacturing, Electronic Test. 21 (2014) 120-122. 